\title{
Computing a largest empty anchored cylinder, and related problems
}

\author{
Frank Follert ${ }^{* \dagger} \quad$ Elmar Schömer* Jürgen Sellen* \\ Michiel Smid ${ }^{\ddagger} \quad$ Christian Thiel ${ }^{\ddagger}$
}

\begin{abstract}
Let $S$ be a set of $n$ points in $\mathbb{R}^{d}$, and let each point $p$ of $S$ have a positive weight $w(p)$. We consider the problem of computing a ray $R$ emanating from the origin (resp. a line $l$ through the origin) such that $\min _{p \in S} w(p) \cdot d(p, R)$ (resp. $\left.\min _{p \in S} w(p) \cdot d(p, l)\right)$ is maximal. If all weights are one, this corresponds to computing a silo emanating from the origin (resp. a cylinder whose axis contains the origin) that does not contain any point of $S$ and whose radius is maximal. For $d=2$, we show how to solve these problems in $O(n \log n)$ time, which is optimal in the algebraic computation tree model. For $d=3$, we give algorithms that are based on the parametric search technique and run in $O\left(n \log ^{4} n\right)$ time. The previous best known algorithms for these three-dimensional problems had almost quadratic running time. In the final part of the paper, we consider some related problems.
\end{abstract}

\section{Introduction}

We consider some problems from the class of facility location problems. These are geometric optimization problems in low-dimensional spaces, and have been widely studied in the literature. (See e.g. $[1,2,4,6,9,11]$.) Before we can state the problems we consider, we need to introduce some notation.

We denote the Euclidean distance between a point $p$ and the origin by $\|p\|$. Also, the Euclidean distance between two points $p$ and $q$ is denoted by $d(p, q)$. If $p$ is a point in $\mathbb{R}^{d}$, and $R$ is a closed subset of $\mathbb{R}^{d}$, then the distance between $p$ and $R$ is defined as $d(p, R):=\min \{d(p, q): q \in R\}$. Finally, we define an anchored ray as a ray that emanates from the origin.

Problem 1 Let $S$ be a set of $n$ points in $\mathbb{R}^{d}$, and let each point $p$ of $S$ have a weight $w(p)$, which is a positive real number. Compute an anchored ray $R$ for which $\min _{p \in S} w(p) \cdot d(p, R)$ is maximal.

*Universität des Saarlandes, Fachbereich 14, Informatik, Lehrstuhl Prof. Hotz, Im Stadtwald, D-66041 Saarbrücken, Germany. E-mail: \{follert, schoemer, sellen\}@cs.uni-sb.de.

†This author was supported by a Graduiertenkolleg Fellowship from DFG, Germany.

Max-Planck-Institut für Informatik, Im Stadtwald, D-66123 Saarbrücken, Germany. E-mail: \{michiel,thiel\}@mpi-sb.mpg.de. These authors were supported by the ESPRIT Basic Research Actions Program, under contract No. 7141 (project ALCOM II). 


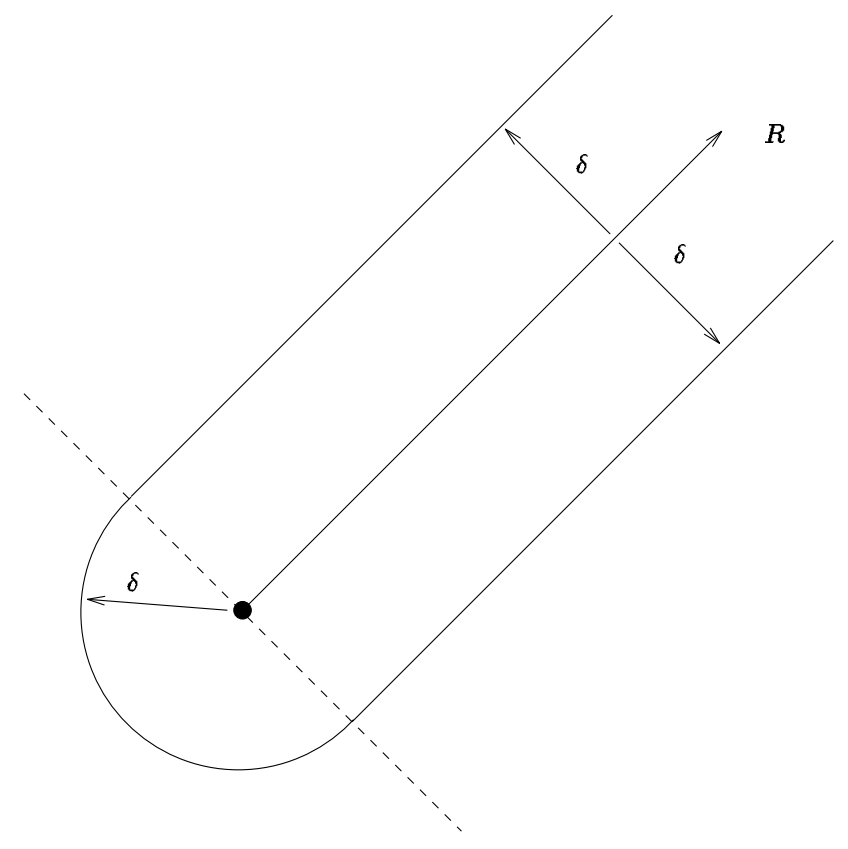

Figure 1: A silo with axis $R$ and radius $\delta$.

We get an obvious generalization if we ask for a line through the origin instead of an anchored ray:

Problem 2 Let $S$ be a set of $n$ points in $\mathbb{R}^{d}$, and let each point $p$ of $S$ have a weight $w(p)$, which is a positive real number. Compute a line l through the origin for which $\min _{p \in S} w(p) \cdot d(p, l)$ is maximal.

Let $R$ be any ray, and let $\delta \geq 0$. The set of all points in $\mathbb{R}^{d}$ that are at distance at most $\delta$ from $R$ is called a silo with axis $R$ and radius $\delta$. (See Figure 1.)

If each point of $S$ has weight one, then Problem 1 asks for the silo whose axis starts in the origin, that does not contain any point of $S$ in its interior, and that has maximal radius. Also, in this case, Problem 2 asks for the cylinder of maximal radius whose axis contains the origin and that does not contain any point of $S$ in its interior.

Problem 1 was posed by Prof. Hotz, and appeared for the first time in Follert's Master Thesis [6]. He shows how to solve this problem in $O(n \alpha(n) \log n)$ time when $d=2$, and in $O\left(n^{2+\epsilon}\right)$ expected time when $d=3$. Here, $\alpha(n)$ denotes the inverse of Ackermann's function, and $\epsilon$ is an arbitrarily small positive constant.

Follert also considers Problem 2. For $d=2$, he shows how to solve this problem in $O(n \log n)$ time. Moreover, he reduces problem Max-Gap-on-a-Circle to Problem 2. (See also Lee and $\mathrm{Wu}[11]$.) Hence, Problem 2 has time complexity $\Omega(n \log n)$ in the algebraic computation tree model. For $d=3$, Follert gives an algorithm that solves Problem 2 in $O\left(n \lambda_{6}(n) \log n\right)$ time, where $\lambda_{6}(n)$ is the maximal length of any Davenport-Schinzel sequence of order six over an alphabet of size $n$. It is known that $\lambda_{6}(n)$ is slightly superlinear. (See Agarwal et al. [3].) Hence, Follert's algorithm has almost quadratic running time. 


\subsection{Our contribution}

In Section 2, we prove some preliminary results. First, we show that we can assume w.l.o.g. that all points have weight one, i.e., it suffices to consider the unit-weight versions of Problems 1 and 2. (This observation appears already in $[6,11]$.) Then we show that the time complexity of Problem 2 is bounded above by that of Problem 1.

In Section 3, we consider the two-dimensional version of Problem 1. We give an extremely simple algorithm that solves this problem in $O(n \log n)$ time. This algorithm uses the lower envelope of some appropriately chosen curves. A careful analysis shows that this lower envelope has linear combinatorial complexity.

The results of Section 2 imply that the two-dimensional version of Problem 2 can also be solved in $O(n \log n)$ time. Since Follert [6] proved an $\Omega(n \log n)$ lower bound for this problem, it follows that our algorithms for solving the planar versions of Problems 1 and 2 are optimal in the algebraic computation tree model.

In Section 4, we consider the three-dimensional version of Problem 1. The appropriate technique to apply seems to be Megiddo's parametric search [12]. We show that this is indeed true. In particular, we show that it suffices to design sequential and parallel algorithms for the following covering problem: Given a set of $n$ disks on the unit sphere, decide whether these disks cover the sphere. Then, Megiddo's technique immediately solves Problem 1.

The overall algorithm for solving Problem 1 has running time $O\left(n \log ^{4} n\right)$. By the results of Section 2, the three-dimensional version of Problem 2 can be solved within the same time bound. Compared with the previous almost quadratic time bounds of [6], these are drastic improvements.

In the final part of Section 4, we give alternative algorithms that solve the covering problem for pseudo disks on the unit sphere.

In Section 5, we consider some related problems. In particular, the dual of the three-dimensional version of Problem 1, which asks for an anchored ray $R$ for which $\max _{p \in S} w(p) \cdot d(p, R)$ is minimal, can be solved in $O\left(n \log ^{4} n\right)$ time using basically the same approach as in Section 4. We also discuss the dual of the three-dimensional version of Problem 2, which seems to be much more difficult. Finally, for $d=3$, we show how to compute a plane $H$ through the origin such that $\max _{p \in S} w(p) \cdot d(p, H)$ is minimal, in $O(n \log n)$ time. It was proved in [11] that the planar version of the latter problem has an $\Omega(n \log n)$ lower bound in the algebraic computation tree model. Hence, our algorithm is optimal in this model.

\section{Some preliminary results}

Let $S$ be a set of points in $\mathbb{R}^{d}$. If $S$ contains the origin, then any anchored ray $R$ (resp. any line $l$ through the origin) is a solution to Problem 1 (resp. 2). Therefore, from now on, we make the following assumption.

Assumption 1 The set $S$ does not contain the origin.

Lemma 1 Let $p=\left(p_{1}, p_{2}, \ldots, p_{d}\right)$ be a point in $\mathbb{R}^{d}$, let $w$ be a positive real number, and let $R$ be an anchored ray in $\mathbb{R}^{d}$. Let $p^{\prime}:=\left(w p_{1}, w p_{2}, \ldots, w p_{d}\right)$. Then $w \cdot d(p, R)=$ $d\left(p^{\prime}, R\right)$. 
Proof: We can assume w.l.o.g. that $R$ is the positive $x_{1}$-axis. First assume that $p_{1} \leq 0$. Then $d(p, R)=\|p\|$ and $d\left(p^{\prime}, R\right)=\left\|p^{\prime}\right\|$, and the claim clearly holds.

Assume that $p_{1}>0$. Let $\alpha$ be the angle between the vector $\vec{p}$ and the ray $R$. Then $\sin \alpha=d(p, R) /\|p\|$ and $\sin \alpha=d\left(p^{\prime}, R\right) /\left\|p^{\prime}\right\|=d\left(p^{\prime}, R\right) /(w\|p\|)$. Hence, $w \cdot d(p, R)=$ $d\left(p^{\prime}, R\right)$

Corollary 1 Let $T(n)$ denote the complexity of the unit-weight version of Problem 1. Then the weighted version of Problem 1 has complexity $O(T(n))$.

Proof: Given a set $S$ of weighted points in $\mathbb{R}^{d}$, the previous lemma shows that we can replace this set by the set $S^{\prime}:=\left\{p^{\prime}: p \in S\right\}$ of unit-weight points.

Lemma 2 Let $T(n)$ be the complexity of Problem 1. Then the complexity of Problem 2 is bounded by $O(T(2 n))$.

Proof: Let $A$ be an algorithm that solves Problem 1 in time $T(n)$. Let $S$ be a set of $n$ points in $\mathbb{R}^{d}$, and let each point $p$ of $S$ have a positive weight $w(p)$. We want to compute a line $l$ through the origin for which $\min _{p \in S} w(p) \cdot d(p, l)$ is maximal.

Let $S^{\prime}:=S \cup-S$, where $-S:=\left\{\left(-p_{1},-p_{2}, \ldots,-p_{d}\right):\left(p_{1}, p_{2}, \ldots, p_{d}\right) \in S\right\}$. We give each point in $-S$ the weight of the corresponding point of $S$. Use algorithm $A$ to compute an anchored ray $R^{*}$ such that $\min _{p \in S^{\prime}} w(p) \cdot d\left(p, R^{*}\right)$ is maximal. Let $l^{*}$ be the line that supports $R^{*}$.

We claim that $l^{*}$ is a solution to Problem 2 for the set $S$. Clearly, this claim proves the lemma. Define $\delta:=\max \left\{\min _{p \in S} w(p) \cdot d(p, l): l\right.$ is a line through the origin $\}$, and $\delta^{*}:=\min _{p \in S} w(p) \cdot d\left(p, l^{*}\right)$. Then we have to prove that $\delta=\delta^{*}$.

It is clear that $\delta \geq \delta^{*}$. Since $d\left(p, l^{*}\right)=\min \left(d\left(p, R^{*}\right), d\left(-p, R^{*}\right)\right)$ for each point $p$, we have $\delta^{*}=\min _{p \in S^{\prime}} w(p) \cdot d\left(p, R^{*}\right)$. Assume that $\delta>\delta^{*}$. Let $l$ be a line through the origin such that $\min _{p \in S} w(p) \cdot d(p, l)>\delta^{*}$, and let $R$ be an anchored ray contained in $l$. Since $d(p, l)=\min (d(p, R), d(-p, R))$ for each point $p$, it follows that

$$
\min _{p \in S^{\prime}} w(p) \cdot d(p, R)=\min _{p \in S} w(p) \cdot d(p, l)>\delta^{*}=\min _{p \in S^{\prime}} w(p) \cdot d\left(p, R^{*}\right)
$$

This is a contradiction, because $R^{*}$ is an optimal anchored ray for the set $S^{\prime}$. This proves that $\delta=\delta^{*}$.

\section{Problem 1: the two-dimensional case}

In this section, we give an optimal algorithm for solving the planar version of Problem 1. This algorithm is obtained by reducing the problem to a simple problem on lower envelopes. We remark that our method has a similarity to those in Melkman and O'Rourke [13] and Agarwal et al.[2]. In order to be self-contained, however, we give all details here. Moreover, we introduce quite some notation. The reason for doing this is to show that the final algorithm is based only on very simple curves.

Let $S$ be a set of $n$ points in the plane, and let each point $p$ of $S$ have a positive weight $w(p)$. We want to compute an anchored ray $R$ such that $\min _{p \in S} w(p) \cdot d(p, R)$ 
is maximal. By Corollary 1, we can assume w.l.o.g. that $w(p)=1$ for all points $p$. Define

$$
\delta^{*}:=\max \left\{\min _{p \in S} d(p, R): R \text { is an anchored ray }\right\} .
$$

Let $\delta_{l}^{*}$ (resp. $\delta_{r}^{*}$ ) denote the analogous quantity where we only consider anchored rays that lie on or to the left (resp. right) of the $y$-axis. It is clear that $\delta^{*}=\max \left(\delta_{l}^{*}, \delta_{r}^{*}\right)$. We show how to compute $\delta_{r}^{*}$. The value $\delta_{l}^{*}$ can be computed in a symmetric way.

Let $\delta_{\text {min }}:=\min \{\|p\|: p \in S\}$. For each $\delta \geq 0$ and each point $p$ of $S$, let $D_{p}^{\delta}$ denote the disk with center $p$ and radius $\delta$. For $0 \leq \delta \leq \delta_{\min }$ and $p \in S$, let $C_{p}^{\delta}$ denote the cone consisting of all anchored rays that intersect or touch the disk $D_{p}^{\delta}$. (Since $\delta \leq \delta_{\min }, D_{p}^{\delta}$ does not contain the origin. Therefore, $C_{p}^{\delta}$ really is a cone.) Note that $C_{p}^{\delta}$ has the origin as its apex.

Observation 1 Using these notations, we have

1. $\delta_{r}^{*}$ is the maximal value of $\delta, 0 \leq \delta \leq \delta_{\min }$, such that there is an anchored ray in the halfplane $x \geq 0$ that does not intersect the interior of any disk $D_{p}^{\delta}, p \in S$.

2. $0 \leq \delta_{r}^{*} \leq \delta_{\min }$.

3. $\delta_{r}^{*}$ is the minimum of $\delta_{\text {min }}$ and the minimal value of $\delta, 0 \leq \delta \leq \delta_{\text {min }}$, such that the cones $C_{p}^{\delta}, p \in S$, cover the halfplane $x \geq 0$.

Let $0 \leq \delta \leq \delta_{\min }$ and let $p \in S$. Consider the intersection of the cone $C_{p}^{\delta}$ with the halfplane $x \geq 0$. Let $I_{p}(\delta)$ be the interval of slopes spanned by all anchored rays that lie in this intersection. We represent each slope by the angle between the ray and the positive $x$-axis. Hence, $I_{p}(\delta) \subseteq[-\pi / 2, \pi / 2]$. We can easily write down this interval explicitly:

Let $p$ have coordinates $\left(p_{1}, p_{2}\right)$, and let $\varphi_{p},-\pi<\varphi_{p} \leq \pi$, be the angle between the vector $\vec{p}$ and the positive $x$-axis. Then, $\sin \varphi_{p}=p_{2} /\|p\|$. Also, for $0 \leq \delta \leq \delta_{\min }$, let $\alpha_{p}^{\delta}$ be the angle between $\vec{p}$ and an anchored ray that is tangent to the disk $D_{p}^{\delta}$. (There are two such tangents, but both define the same angle.) Then, $0 \leq \alpha_{p}^{\delta} \leq \pi / 2$ and $\sin \alpha_{p}^{\delta}=\delta /\|p\|$. (See Figure 2.) If $p_{1} \geq 0$, then

$$
I_{p}(\delta)= \begin{cases}{\left[\varphi_{p}-\alpha_{p}^{\delta}, \varphi_{p}+\alpha_{p}^{\delta}\right]} & \text { if } 0 \leq \delta \leq \delta_{\min } \text { and } \delta \leq p_{1} \\ {\left[\varphi_{p}-\alpha_{p}^{\delta}, \pi / 2\right]} & \text { if } p_{1} \leq \delta \leq \delta_{\min } \text { and } p_{2} \geq \mathbf{0} \\ {\left[-\pi / 2, \varphi_{p}+\alpha_{p}^{\delta}\right]} & \text { if } p_{1} \leq \delta \leq \delta_{\min } \text { and } p_{2} \leq \mathbf{0}\end{cases}
$$

If $p_{1} \leq 0$, then

$$
I_{p}(\delta)= \begin{cases}\emptyset & \text { if } 0 \leq \delta \leq \delta_{\min } \text { and } \delta \leq-p_{1} \\ {\left[\varphi_{p}-\alpha_{p}^{\delta}, \pi / 2\right]} & \text { if }-p_{1} \leq \delta \leq \delta_{\min } \text { and } p_{2} \geq 0 \\ {\left[-\pi / 2, \varphi_{p}+\alpha_{p}^{\delta}\right]} & \text { if }-p_{1} \leq \delta \leq \delta_{\min } \text { and } p_{2} \leq 0\end{cases}
$$

It is clear that the cones $C_{p}^{\delta}, p \in S$, cover the halfplane $x \geq 0$ if and only if the intervals $I_{p}(\delta), p \in S$, cover $[-\pi / 2, \pi / 2]$. Hence, $\delta_{r}^{*}$ is the minimum of

1. $\delta_{\min }$, and 


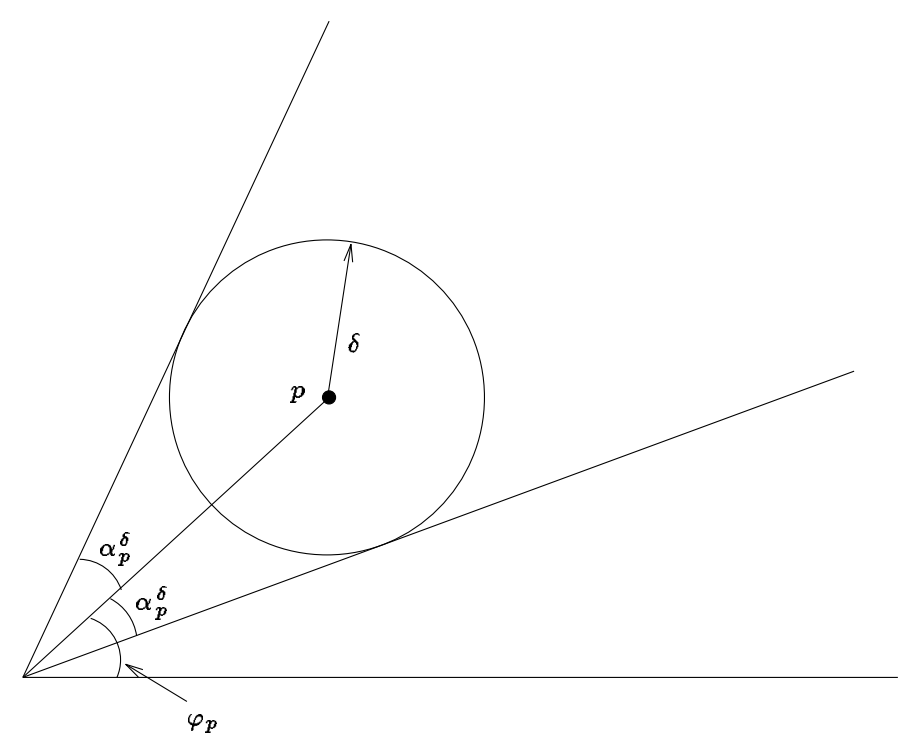

Figure 2: Illustration of the angles $\varphi_{p}$ and $\alpha_{p}^{\delta}$.

2. the minimal value of $\delta, 0 \leq \delta \leq \delta_{\min }$, such that the intervals $I_{p}(\delta)$ cover $[-\pi / 2, \pi / 2]$.

Using the intervals $I_{p}(\delta)$ has the disadvantage that we need non-algebraic functions. In order to stay within the algebraic computation tree model, our algorithm works with the intervals

$$
J_{p}(\delta):=\sin \left(I_{p}(\delta)\right)=\left\{\sin \gamma: \gamma \in I_{p}(\delta)\right\} .
$$

Note that $I_{p}(\delta) \subseteq[-\pi / 2, \pi / 2]$ and that the function $\sin (\cdot)$ is increasing on $[-\pi / 2, \pi / 2]$. Using the relations $\sin \varphi_{p}=p_{2} /\|p\|, \cos \varphi_{p}=p_{1} /\|p\|, \sin \alpha_{p}^{\delta}=\delta /\|p\|, \cos \alpha_{p}^{\delta}=$ $\sqrt{p_{1}^{2}+p_{2}^{2}-\delta^{2}} /\|p\|$, and $\sin (x+y)=\sin x \cos y+\cos x \sin y$, we get the following expressions for $J_{p}(\delta)$. If $p_{1} \geq 0$, then

$$
J_{p}(\delta)= \begin{cases}{\left[\frac{p_{2} \sqrt{p_{1}^{2}+p_{2}^{2}-\delta^{2}}-p_{1} \delta}{\|p\|^{2}}, \frac{p_{2} \sqrt{p_{1}^{2}+p_{2}^{2}-\delta^{2}}+p_{1} \delta}{\|p\|^{2}}\right]} & \text { if } 0 \leq \delta \leq \delta_{\text {min }} \text { and } \delta \leq p_{1}, \\ {\left[\frac{p_{2} \sqrt{p_{1}^{2}+p_{2}^{2}-\delta^{2}}-p_{1} \delta}{\|p\|^{2}}, 1\right]} & \text { if } p_{1} \leq \delta \leq \delta_{\text {min }} \text { and } p_{2} \geq 0 \\ {\left[-1, \frac{p_{2} \sqrt{p_{1}^{2}+p_{2}^{2}-\delta^{2}}+p_{1} \delta}{\|p\|^{2}}\right]} & \text { if } p_{1} \leq \delta \leq \delta_{\text {min }} \text { and } p_{2} \leq \mathbf{0} .\end{cases}
$$

If $p_{1} \leq 0$, then

$$
J_{p}(\delta)= \begin{cases}\emptyset & \text { if } 0 \leq \delta \leq \delta_{\min } \text { and } \delta \leq-p_{1} \\ {\left[\frac{p_{2} \sqrt{p_{1}^{2}+p_{2}^{2}-\delta^{2}}-p_{1} \delta}{\|p\|^{2}}, 1\right]} & \text { if }-p_{1} \leq \delta \leq \delta_{\min } \text { and } p_{2} \geq 0 \\ {\left[-1, \frac{p_{2} \sqrt{p_{1}^{2}+p_{2}^{2}-\delta^{2}}+p_{1} \delta}{\|p\|^{2}}\right]} & \text { if }-p_{1} \leq \delta \leq \delta_{\min } \text { and } p_{2} \leq \mathbf{0}\end{cases}
$$

The value of $\delta_{r}^{*}$ is equal to the minimum of $\delta_{\min }$ and the minimal value of $\delta, 0 \leq \delta \leq$ $\delta_{\text {min }}$, such that the intervals $J_{p}(\delta)$ cover $[-1,1]$. 
For $p \in S$, let

$$
R_{p}:=\left\{(x, \delta): 0 \leq \delta \leq \delta_{\min }, x \in J_{p}(\delta)\right\} .
$$

The region $R_{p}$ is contained in the rectangle $[-1,1] \times\left[0, \delta_{\text {min }}\right]$.

Observation $2 \delta_{r}^{*}$ is the minimum of

1. $\delta_{\text {min }}$, and

2. the minimal value of $\delta, 0 \leq \delta \leq \delta_{\text {min }}$, such that the horizontal segment with endpoints $(-1, \delta)$ and $(1, \delta)$ is completely contained in $\bigcup_{p \in S} R_{p}$.

Let $l_{p}$ be the lower envelope of $R_{p}$. Then, $l_{p}$ is the graph of a continuous function on a subinterval of $[-1,1]$. Finally, let $L$ be the lower envelope of the graphs $l_{p}, p \in S$, and the line segment with endpoints $\left(-1, \delta_{\min }\right)$ and $\left(1, \delta_{\min }\right)$.

Observation $3 \delta_{r}^{*}$ is the $y$-coordinate of a highest vertex of $L$.

We now analyze the lower envelope $L$. Let $B_{l}, B_{r}, B_{t}$ and $B_{b}$ be the left, right, top and bottom side of the rectangle $[-1,1] \times\left[0, \delta_{\text {min }}\right]$, respectively.

Let $p=\left(p_{1}, p_{2}\right)$ be a point of $S$, and consider the graph $l_{p}$. If $p_{1} \geq 0$, then $l_{p}$ consists of a decreasing part $l_{p}^{-}$that has $\left(p_{2} /\|p\|, 0\right)$ as its lowest and rightmost endpoint, and an increasing part $l_{p}^{+}$that has $\left(p_{2} /\|p\|, 0\right)$ as its lowest and leftmost endpoint. Moreover, $l_{p}^{-}$(resp. $l_{p}^{+}$) has its leftmost (resp. rightmost) endpoint on $B_{l}$ or $B_{t}$ (resp. $B_{r}$ or $B_{t}$ ). If $p_{1} \leq 0$ and $p_{2} \geq 0$, then $l_{p}$ is decreasing from some point on $B_{t}$ to some point on $B_{r}$. Finally, if $p_{1} \leq 0$ and $p_{2} \leq 0$, then $l_{p}$ is increasing from some point on $B_{l}$ to some point on $B_{t}$.

Let $p=\left(p_{1}, p_{2}\right)$ and $q=\left(q_{1}, q_{2}\right)$ be two distinct points of $S$. We claim that the graphs $l_{p}$ and $l_{q}$ intersect at most twice. First, we give a geometric explanation for this claim. Then, in Lemma 3 below, we give a rigorous proof.

For the intuitive explanation, assume that $p_{1}$ and $q_{1}$ are both positive and that $\varphi_{q}>\varphi_{p}$. For $0 \leq \delta \leq \delta_{\text {min }}$, let $U_{p}(\delta)$ (resp. $L_{p}(\delta)$ ) be the anchored ray that is upper (resp. lower) tangent to the disk $D_{p}^{\delta}$. Define $U_{q}(\delta)$ and $L_{q}(\delta)$ analogously.

Intersections of $l_{p}$ and $l_{q}$ are in one-to-one correspondence with values of $\delta$ such that $\left\{U_{p}(\delta), L_{p}(\delta)\right\} \cap\left\{U_{q}(\delta), L_{q}(\delta)\right\} \neq \emptyset$.

Consider what happens when we grow $\delta$ from 0 to $\delta_{\min }$. Initially, $U_{p}(\delta)=L_{p}(\delta)$ and $U_{q}(\delta)=L_{q}(\delta)$. If $\delta$ increases, then the tangents $U_{p}(\delta)$ and $L_{p}(\delta)$ move in opposite directions. Similarly, the tangents $U_{q}(\delta)$ and $L_{q}(\delta)$ move in opposite directions. (See Figure 3.) Clearly, there is exactly one $\delta_{0}$ such that $L_{q}\left(\delta_{0}\right)=U_{p}\left(\delta_{0}\right)$. This corresponds to an intersection of $l_{q}^{-}$and $l_{p}^{+}$. Also, for $\delta<\delta_{0}$, there are no intersections between $l_{p}$ and $l_{q}$. Now we grow $\delta$ further, from $\delta_{0}$ to the next "time" $\delta_{1}$ at which $\left\{U_{p}\left(\delta_{1}\right), L_{p}\left(\delta_{1}\right)\right\} \cap$ $\left\{U_{q}\left(\delta_{1}\right), L_{q}\left(\delta_{1}\right)\right\} \neq \emptyset$. (If there is no such time, then the graphs $l_{p}$ and $l_{q}$ intersect exactly once, and we are done.) Then, $U_{p}\left(\delta_{1}\right)=U_{q}\left(\delta_{1}\right)$ or $L_{p}\left(\delta_{1}\right)=L_{q}\left(\delta_{1}\right)$. Assume w.l.o.g. that at time $\delta_{1}, U_{p}\left(\delta_{1}\right)=U_{q}\left(\delta_{1}\right)$. This corresponds to the second intersection between $l_{p}$ and $l_{q}$; more precisely, an intersection between $l_{p}^{+}$and $l_{q}^{+}$. Note that then $U_{p}(\delta)$ must move faster than $U_{q}(\delta)$. Hence, for $\delta>\delta_{1}$, these two tangents never coincide any more. That is, $l_{p}^{+}$and $l_{q}^{+}$intersect only once. Now look at $L_{p}(\delta)$ and 


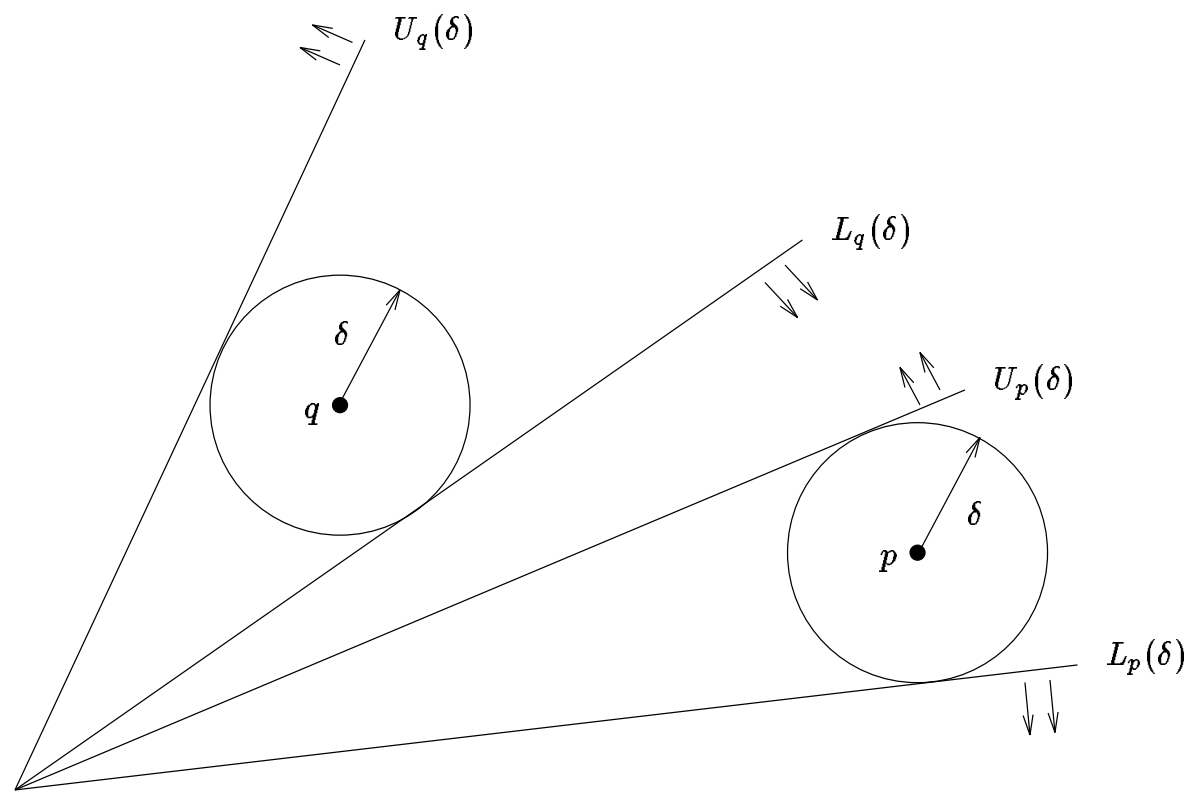

Figure 3: Growing $\delta$ from 0 to $\delta_{\min }$.

$L_{q}(\delta)$ : Since $L_{p}(\delta)$ and $U_{p}(\delta)$ (resp. $L_{q}(\delta)$ and $U_{q}(\delta)$ ) move at the same, but opposite, velocities, $L_{q}(\delta)$ will never overtake $L_{p}(\delta)$. That is, $l_{p}^{-}$and $l_{q}^{-}$do not intersect.

This concludes the intuition why the graphs $l_{p}$ and $l_{q}$ intersect at most twice. We now prove this rigorously.

Lemma 3 Let $p$ and $q$ be two distinct points of $S$. Then, the graphs $l_{p}$ and $l_{q}$ intersect at most twice.

Proof: Assume first that $\varphi_{p}=\varphi_{q}$. Then, $\|p\| \neq\|q\|$. This implies that for all $\delta$, $0 \leq \delta \leq \delta_{\text {min }}$, the cone $C_{p}^{\delta}$ is completely contained inside $C_{q}^{\delta}$, or vice versa. As a result, $l_{p}$ and $l_{q}$ have only one intersection point, with $y$-coordinate zero.

Assume from now on that $\varphi_{p} \neq \varphi_{q}$. W.l.o.g. assume that $\varphi_{q}>\varphi_{p}$. Let $I$ be the interval of all values $\delta$ such that $J_{p}(\delta)$ and $J_{q}(\delta)$ are both non-empty. Consider the function

$$
f(\delta):=\varphi_{q}-\varphi_{p}-\alpha_{q}^{\delta}-\alpha_{p}^{\delta},
$$

for $\delta \in I$. Then $f(\delta)=0$ if and only if the increasing part of $l_{p}$ and the decreasing part of $l_{q}$ have an intersection point with $y$-coordinate $\delta$. Note that

$$
f(\delta)=\varphi_{q}-\varphi_{p}-\arcsin (\delta /\|q\|)-\arcsin (\delta /\|p\|) .
$$

The derivative of $f$ is equal to

$$
f^{\prime}(\delta)=\frac{-1}{\sqrt{\|q\|^{2}-\delta^{2}}}+\frac{-1}{\sqrt{\|p\|^{2}-\delta^{2}}} .
$$

Hence, $f^{\prime}$ is strictly negative, which implies that $f$ has at most one root. 
Next let

$$
g(\delta):=\varphi_{q}-\varphi_{p}+\alpha_{q}^{\delta}-\alpha_{p}^{\delta},
$$

for $\delta \in I$. The roots of $g$ are in one-to-one correspondence with the intersections between the increasing parts of $l_{p}$ and $l_{q}$. We have

$$
g^{\prime}(\delta)=\frac{1}{\sqrt{\|q\|^{2}-\delta^{2}}}-\frac{1}{\sqrt{\|p\|^{2}-\delta^{2}}} .
$$

If $\|p\|=\|q\|$, then $g(\delta)=\varphi_{q}-\varphi_{p}$, which is never zero. If $\|p\| \neq\|q\|$, then $g^{\prime}$ is either strictly positive or strictly negative for all $\delta \in I$. Hence, the function $g$ has at most one root.

In a completely symmetric way, it follows that the function

$$
h(\delta):=\varphi_{q}-\varphi_{p}-\alpha_{q}^{\delta}+\alpha_{p}^{\delta},
$$

for $\delta \in I$ has at most one root. That is, the decreasing parts of $l_{p}$ and $l_{q}$ intersect at most once.

Now we can prove the lemma. First assume that $p_{1} \leq 0$. Then $l_{p}$ consists only of a decreasing part, or only of an increasing part. If $q_{1} \leq 0$, then $l_{q}$ consists of one monotone part. The above analysis shows that in this case, $l_{p}$ and $l_{q}$ intersect at most once. If $q_{1}>0$, then $l_{q}$ consists of two monotone parts, each of which intersects $l_{p}$ at most once. Hence, in this case, $l_{p}$ and $l_{q}$ intersect at most twice.

If $p_{1} \geq 0$ and $q_{1} \leq 0$, then a symmetric argument shows that $l_{p}$ and $l_{q}$ intersect at most twice.

It remains to consider the case where $p_{1}>0$ and $q_{1}>0$. We proved above that the increasing part $l_{p}^{+}$of $l_{p}$ and the decreasing part $l_{q}^{-}$of $l_{q}$ intersect at most once.

Assume that $l_{p}^{+}$and $l_{q}^{+}$intersect. Then the analysis above shows that they intersect exactly once. Since the function $g$ is monotone, $g(0)=\varphi_{q}-\varphi_{p}>0$, and $g$ has a root, this function is decreasing. But this implies that $h$ is increasing. Since $h(0)>0$, the function $h$ does not have any root, which proves that $l_{p}^{-}$and $l_{q}^{-}$do not intersect.

If $l_{p}^{-}$and $l_{q}^{-}$intersect, then it follows in a completely symmetric way that $l_{p}^{+}$and $l_{q}^{+}$do not intersect.

This proves that $l_{p}$ and $l_{q}$ intersect at most twice.

Lemma 4 The lower envelope $L$ consists of $O(n)$ vertices.

Proof: We will show that the names of the points that correspond to the edges of $L$, when we traverse $L$ from left to right, form a Davenport-Schinzel sequence of order two. This will prove the claim. (See e.g. [8].) Hence, we must show that for any pair $p$ and $q$ of distinct points of $S$, this sequence of names does not contain a subsequence of the form $p \ldots q \ldots p \ldots q$. But this follows from the fact that $l_{p}$ and $l_{q}$ intersect at most twice, and from the restrictions on the endpoint of these graphs.

Now we are ready to give the algorithm for computing $\delta_{r}^{*}$ and a corresponding ray $R^{*}$.

1. Compute the graphs $l_{p}, p \in S$. 
2. Compute the lower envelope $L$ of the graphs $l_{p}, p \in S$, and the horizontal segment with endpoints $\left(-1, \delta_{\min }\right)$ and $\left(1, \delta_{\min }\right)$.

3. Walk along $L$ and find a highest vertex on it. Let this vertex have coordinates $(a, \delta)$.

4. Output $\delta$ and the anchored ray $R:=\left\{\left(x, a x / \sqrt{1-a^{2}}\right): x \geq 0\right\}$.

To prove the correctness of this algorithm, consider the vertex $(a, \delta)$ that is found in Step 3. Observation 3 implies that $\delta=\delta_{r}^{*}$. Let $\varphi$ be the angle such that $-\pi / 2 \leq$ $\varphi \leq \pi / 2$ and $\sin \varphi=a$. Let $R^{*}$ be the anchored ray that makes an angle of $\varphi$ with the positive $x$-axis. Then $\delta=\min _{p \in S} d\left(p, R^{*}\right)$. It is easy to see that $R=R^{*}$.

Next we analyze the running time of our algorithm. Step 1 takes $O(n)$ time. The lower envelope $L$ can be computed by a divide-and-conquer algorithm. (See e.g. [8].) Since $L$ has linear size, this algorithm, and hence Step 2, takes $O(n \log n)$ time. Step 3 takes $O(n)$ time, and Step 4 takes $O(1)$ time.

We have proved the following result.

Theorem 1 Let $S$ be a set of $n$ points in the plane, and let each point $p$ of $S$ have a positive weight $w(p)$. In $O(n \log n)$ time, we can compute an anchored ray $R^{*}$ for which $\min _{p \in S} w(p) \cdot d\left(p, R^{*}\right)$ is maximal.

Lemma 2 immediately implies the following result.

Corollary 2 Let $S$ be a set of $n$ points in the plane, and let each point $p$ of $S$ have a positive weight $w(p)$. In $O(n \log n)$ time, we can compute a line $l^{*}$ through the origin for which $\min _{p \in S} w(p) \cdot d\left(p, l^{*}\right)$ is maximal.

The results of Theorem 1 and Corollary 2 are optimal in the algebraic computation tree model. (Note that our algorithm fits into this model.) Follert [6] proves an $\Omega(n \log n)$ lower bound for Problem 2. It follows from Lemma 2 that this lower bound holds for Problem 1 as well.

\section{Problem 1: the three-dimensional case}

\subsection{The parametric search technique}

We briefly recall Megiddo's parametric search technique [12]. (See also [4].)

Suppose we are given a fixed set of $n$ data items, such as points in $\mathbb{R}^{3}$. Let $\mathcal{P}(t)$ be a decision problem whose value depends on the $n$ data items and a real parameter $t$. Assume that $\mathcal{P}$ is monotone, meaning that if $\mathcal{P}\left(t_{0}\right)$ is true for some $t_{0}$, then $\mathcal{P}(t)$ is also true for all $t<t_{0}$. Our aim is to find the maximal value of $t$ for which $\mathcal{P}(t)$ is true. We denote this value by $t^{*}$.

Assume we have a sequential algorithm $A_{s}$ that, given the $n$ data items and $t$, decides if $\mathcal{P}(t)$ is true or not. The control flow of this algorithm is governed by comparisons, each of which involves testing the sign of some low-degree polynomial in $t$. Let $T_{s}$ and $C_{s}$ denote the running time and the number of comparisons made by 
algorithm $A_{s}$, respectively. Note that by running $A_{s}$ on input $t$, we can decide if $t \leq t^{*}$ or $t>t^{*}$ : we have $t \leq t^{*}$ iff $\mathcal{P}(t)$ is true.

The parametric search technique simulates $A_{s}$ on the unknown value $t^{*}$. Whenever $A_{s}$ reaches a branching point that depends on a comparison operation, the comparison can be reduced to testing the sign of a suitable low-degree polynomial $f(t)$ at $t=t^{*}$. The algorithm computes the roots of this polynomial and checks each root $a$-by running $A_{s}$ on input $a$-to see if it is less than or equal to $t^{*}$. In this way, the algorithm identifies two successive roots between which $t^{*}$ must lie and thus determines the sign of $f\left(t^{*}\right)$. Hence, we get an interval $I$ that contains $t^{*}$. Also the comparison now being resolved, the execution can proceed. As we proceed through the execution, each comparison that we resolve results in constraining $I$ further and we get a sequence of progressively smaller intervals each known to contain $t^{*}$. The simulation will run to completion and we are left with an interval $I$ that contains $t^{*}$. It can be shown that for any real number $r \in I, \mathcal{P}(r)$ is true. Therefore, $t^{*}$ must be the right endpoint of $I$.

Since $A_{s}$ makes at most $C_{s}$ comparisons during its execution, the entire simulation and, hence, the computation of $t^{*}$ take $O\left(C_{s} T_{s}\right)$ time. To speed up this algorithm, Megiddo replaces $A_{s}$ by a parallel algorithm $A_{p}$ that uses $P$ processors and runs in $T_{p}$ parallel time. At each parallel step, let $A_{p}$ make a maximum of $W_{p}$ independent comparisons. Then our algorithm simulates $A_{p}$ sequentially, again at the unknown value $t^{*}$. At each parallel step, we get at most $W_{p}$ low-degree polynomials in $t$. We compute the roots of all of them and do a binary search among them using repeated median finding to make the probes for $t^{*}$. For each probe, we run the sequential algorithm $A_{s}$. In this way, we get the correct sign of each polynomial in $t^{*}$, and our algorithm can simulate the next parallel step of $A_{p}$.

For the simulation of each parallel step, we spend $O\left(W_{p}\right)$ time for median finding. Hence, the entire simulation of this step takes time $O\left(W_{p}+T_{s} \log W_{p}\right)$. As a result, the entire algorithm computes $t^{*}$ in time $O\left(W_{p} T_{p}+T_{s} T_{p} \log W_{p}\right)$. Since $W_{p} \leq P$, the running time is bounded by $O\left(P T_{p}+T_{s} T_{p} \log P\right)$.

\subsection{Applying the parametric search technique}

Let $S$ be a set of $n$ points in $\mathbb{R}^{3}$. Each point $p$ of $S$ has a positive weight $w(p)$. Define

$$
\delta^{*}:=\max \left\{\min _{p \in S} w(p) \cdot d(p, R): R \text { is an anchored ray }\right\}
$$

Our goal is to compute $\delta^{*}$ together with the corresponding ray $R^{*}$. We saw already that we may assume w.l.o.g. that $w(p)=1$ for all points $p$.

The way we apply parametric search is standard by now. (See e.g.[2].) We have to solve the following decision problem $\mathcal{P}(\delta)$ : Given the set $S$ and the real number $\delta \geq 0$, decide if there is an anchored ray $R$ such that $\min _{p \in S} d(p, R) \geq \delta$. It is clear that $\mathcal{P}$ is monotone, and $\delta^{*}$ is the maximal $\delta$ for which $\mathcal{P}(\delta)$ is true.

We reformulate the decision problem $\mathcal{P}(\delta)$ in the following way. Let $\delta \geq 0$. For each point $p$ of $S$, let $B_{p}^{\delta}$ denote the ball with center $p$ and radius $\delta$. Then $\mathcal{P}(\delta)$ is true if and only if there is an anchored ray $R$ that does not intersect the interior of any of these balls.

Let $\delta_{\text {min }}:=\min \{\|p\|: p \in S\}$. Then $\mathcal{P}(\delta)$ is clearly false for $\delta>\delta_{\min }$. 
For $0 \leq \delta \leq \delta_{\min }$, let $C_{p}^{\delta}$ denote the circular cone consisting of all anchored rays that intersect or touch the ball $B_{p}^{\delta}$. This cone intersects the unit sphere-i.e., the surface of the ball of radius one centered at the origin-in a disk. We denote this disk by $D_{p}^{\delta}$.

Let $0 \leq \delta \leq \delta_{\min }$. It is clear that $\mathcal{P}(\delta)$ is true if and only if there is a point $x$ on the unit sphere that is not contained in the interior of any of these $n$ disks. If there is such a point $x$, then the ray $R$ that starts in the origin and contains $x$ satisfies $\min _{p \in S} d(p, R) \geq \delta$. In other words, $\mathcal{P}(\delta)$ is true if and only if the interiors of these $n$ disks do not cover the unit sphere.

\subsubsection{Deciding the covering problem using a convex hull algorithm}

Consider the $n$ disks $D_{p}^{\delta}, p \in S$, on the unit sphere $\mathcal{S}^{2}$. We want to decide if the interiors of these disks cover $\mathcal{S}^{2}$.

Let $I_{p}$ (resp. $\gamma_{p}$ ) denote the interior (resp. boundary) of $D_{p}^{\delta}, p \in S$, and let $I:=$ $\bigcup_{p \in S} I_{p}$. Let $H_{p}$ denote the plane whose intersection with the unit sphere $\mathcal{S}^{2}$ is equal to $\gamma_{p}$. Let $H_{p}^{+}$denote the closed halfspace bounded by $H_{p}$ containing the origin. The intersection $P o l:=\cap_{p \in S} H_{p}^{+}$of these halfspaces is a possibly unbounded convex polyhedron. Every point of $\mathcal{S}^{2}$ contained in the union $I$ is not contained in at least one of the halfspaces $H_{p}^{+}$, and hence not in $P o l$. Every point in $\mathcal{S}^{2}-I$ is contained in every halfspace $H_{p}^{+}$and hence in $P o l$. Consequently, the intersection of the polyhedron Pol and the unit sphere $\mathcal{S}^{2}$ corresponds exactly to the set of admissible orientations, i.e., the set of all ray orientations maintaining Euclidean distance $\geq \delta$ to all points $p \in S$. Therefore, $\mathcal{P}(\delta)$ is true iff $P$ ol $\cap \mathcal{S}^{2}$ is not empty.

Since all halfspaces $H_{p}^{+}$contain the origin, this is also true for Pol, and its construction can be reduced to a $3 \mathrm{D}$ convex hull problem by a standard dual transformation. The convex hull of $n$ points in 3 -space can be computed in $O(n \log n)$ sequential time and in $O\left(\log ^{2} n\right)$ parallel time using $n$ processors on a CREW PRAM. (See $[5,15]$.)

We remark that it is not necessary to compute the intersection of Pol and $\mathcal{S}^{2}$ explicitly. Pol $\cap \mathcal{S}^{2}$ is empty if and only if $P o l$ is bounded and all its vertices are contained in the interior of the unit ball.

The discussion above together with the results of Section 4.1 immediately provide a solution for Problem 1:

Theorem 2 Let $S$ be a set of $n$ points in $\mathbb{R}^{3}$, and let each point $p$ of $S$ have a positive weight $w(p)$. In $O\left(n \log ^{4} n\right)$ time, we can compute an anchored ray $R^{*}$ for which $\min _{p \in S} w(p) \cdot d\left(p, R^{*}\right)$ is maximal.

Corollary 3 Let $S$ be a set of $n$ points in $\mathbb{R}^{3}$, and let each point $p$ of $S$ have a positive weight $w(p)$. In $O\left(n \log ^{4} n\right)$ time, we can compute a line $l^{*}$ through the origin for which $\min _{p \in S} w(p) \cdot d\left(p, l^{*}\right)$ is maximal.

\subsection{Solving the covering problem for pseudo disks}

The algorithm we gave in Section 4.2.1 only works for disks. In this section, we give an alternative algorithm that works for pseudo disks on the unit sphere. Using this 
alternative algorithm for solving Problem 1 would lead to a running time of $O\left(n \log ^{5} n\right)$. We include the present section, however, because it is more general, and, hence, may have applications for other problems.

Let $D_{1}, D_{2}, \ldots, D_{n}$ be a set of $n$ pseudo disks on the unit sphere. That is, for $i \neq j$, the boundaries of $D_{i}$ and $D_{j}$ intersect at most twice. We assume that the boundary of each $D_{i}$ is a closed curve that can be described by a constant number of polynomials, each having a degree that is bounded by a constant. We want to decide if the interiors of these pseudo disks cover the unit sphere. Clearly, we can use the arrangement of the pseudo disks for deciding this. This arrangement, however, may have size $\Omega\left(n^{2}\right)$. In the next two sections, we give sequential and parallel algorithms that solve this covering problem much more efficiently.

\subsubsection{A sequential algorithm that decides the covering problem}

Let $I_{i}$ (resp. $\gamma_{i}$ ) denote the interior (resp. boundary) of $D_{i}, 1 \leq i \leq n$, and let $I:=\bigcup_{i=1}^{n} I_{i}$. (Note that there may be $i \neq j$ such that $\gamma_{i}=\gamma_{j}$.) We denote the closure of $I$ by $\operatorname{cl}(I)$. The boundary $B$ of $I$ is equal to

$$
B=\operatorname{cl}(I) \backslash I=\left(\bigcup_{i=1}^{n} D_{i}\right) \backslash\left(\bigcup_{i=1}^{n} I_{i}\right) .
$$

The interiors of the pseudo disks $D_{1}, D_{2}, \ldots, D_{n}$ cover the unit sphere if and only if $B$ is empty. Hence, our problem can be solved by computing the boundary $B$ rather than the entire arrangement of the $n$ pseudo disks.

The boundary $B$ is a planar graph on the unit sphere. Each edge of this graph is part of a curve $\gamma_{i}$ for some $i$, and each vertex is an intersection point of at least two distinct curves. We choose an arbitrary point $p_{i}$ on each curve $\gamma_{i}, 1 \leq i \leq n$, with the restriction that $p_{i}=p_{j}$ if $\gamma_{i}=\gamma_{j}$. Then, if $\gamma_{i}$ does not intersect any other curve, it forms an edge of $B$ with both endpoints equal to $p_{i}$. Note that $B$ can have isolated vertices: If three curves intersect in one point $x$, and there is an arbitrarily small disk $\alpha$ (not equal to any of $D_{1}, D_{2}, \ldots, D_{n}$ ) centered at $x$ such that $\alpha \backslash\{x\}$ is contained in the union of the interiors of these three curves, then $x$ is a vertex of $B$, and $x$ is not incident to any edge of $B$.

The proof of the following lemma can be found in Kedem et al. [10]. (See also Remark (2) on page 66 in [10].)

Lemma 5 ([10]) The boundary $B$ is a planar graph on the unit sphere, and, if $n \geq 3$, it contains at most $6 n-12$ vertices.

In [10], an algorithm is given that computes the boundary of the union of $n$ regions in the plane, each of which is bounded by a simple closed Jordan curve. This algorithm follows the divide-and-conquer paradigm, and the merge step is implemented by using a plane sweep algorithm of Ottmann, Widmayer and Wood [14] for computing the boundary of the union of superimposed polygonal planar regions. This plane sweep algorithm also works if the edges of the planar regions are curved. We can easily modify this algorithm such that it computes the boundary $B$ : 
Consider the pseudo disks $D_{1}, D_{2}, \ldots, D_{n}$. Recursively compute the boundary $B_{1}$ (resp. $B_{2}$ ) of the union of the interiors of $D_{1}, D_{2}, \ldots, D_{n / 2}\left(\operatorname{resp} . D_{n / 2+1}, D_{n / 2+2}, \ldots, D_{n}\right)$. Note that $B_{1}$ and $B_{2}$ are planar graphs on the unit sphere. Let $l$ and $r$ be the points on the unit sphere with minimal and maximal $y$-coordinate, respectively. Using the algorithm of [14], we compute the boundary $B$ from $B_{1}$ and $B_{2}$ by sweeping a circular arc with endpoints $l$ and $r$ around the unit sphere. Let $b_{1}$ and $b_{2}$ denote the number of edges of $B_{1}$ and $B_{2}$, respectively, and let $t$ denote the number of intersections between $B_{1}$ and $B_{2}$. Then this sweep algorithm runs in time $O\left(\left(b_{1}+b_{2}+t\right) \log \left(b_{1}+b_{2}\right)\right)$. It follows from Lemma 5 that $b_{1}+b_{2}=O(n)$. Since each intersection point between $B_{1}$ and $B_{2}$ is a vertex of $B$, Lemma 5 also implies that $t=O(n)$. Hence, the entire sweep algorithm runs in time $O(n \log n)$. This shows that the entire divide-and-conquer algorithm for computing the boundary $B$ takes $O\left(n \log ^{2} n\right)$ time. The interiors of the input pseudo disks $D_{1}, D_{2}, \ldots, D_{n}$ cover the unit sphere if and only if the graph $B$ is empty. If $B$ is not empty, then any vertex of $B$ is a point on the unit sphere that is not contained in the interior of any pseudo disk. We have proved the following result.

Theorem 3 Let $D_{1}, D_{2}, \ldots, D_{n}$ be a set of pseudo disks on the unit sphere. In $O\left(n \log ^{2} n\right)$ time, we can decide if the union of the interiors of these pseudo disks covers the unit sphere. If this is not the case, then the algorithm finds a point on the unit sphere that is not contained in the interior of any pseudo disk.

\subsubsection{A parallel algorithm that decides the covering problem}

Now we give a parallel algorithm for computing the boundary $B$. Consider again the pseudo disks $D_{1}, D_{2}, \ldots, D_{n}$. The algorithm uses $n$ processors. The first (resp. last) $n / 2$ processors compute the boundary $B_{1}$ (resp. $B_{2}$ ) of the union of the interiors of $D_{1}, D_{2}, \ldots, D_{n / 2}\left(\operatorname{resp} . D_{n / 2+1}, D_{n / 2+2}, \ldots, D_{n}\right)$. It remains to describe the merge step. That is, given $B_{1}$ and $B_{2}$, how to compute the boundary $B$ of the union of the interiors of the $n$ input pseudo disks.

Rüb [16] gives a parallel algorithm based on a segment tree, that computes the intersections among red and blue curved segments in the plane. The interiors of the red (resp. blue) segments are assumed to be pairwise disjoint. Also, each segment is assumed to be $x$-monotone, meaning that any vertical line intersects a segment at most once. Finally, it is assumed that each red-blue pair of segments intersect at most a constant number of times. If $n$ denotes the total number of red and blue segments, and $t$ denotes the total number of intersection points among the red-blue pairs of segments, then Rüb's algorithm runs on a CREW-PRAM in time $O(\log n+t / n)$ using $n$ processors.

This algorithm can be used to compute the boundary $B$ from $B_{1}$ and $B_{2}$ : In our case, the slabs that define the segment tree are bounded by circular arcs on the unit sphere with two fixed diametral endpoints. In order to guarantee that each curved edge of $B_{1}$ and $B_{2}$ is monotone, we cut each of them into at most two parts. Note that, by Lemma $5, t=O(n)$. Hence, using Rüb's algorithm, we compute all intersections of $B_{1}$ and $B_{2}$ in $O(\log n)$ time using $n$ processors.

Then, for each edge $e$ of $B_{1}$, we sort the intersection points on this edge. This gives the arrangement $A$ of the union $B_{1}$ and $B_{2}$. Given this arrangement, we compute the 
boundary $B$ by removing the appropriate vertices and edges from $A$. All this can be done in $O(\log n)$ time using $n$ processors.

Hence, the entire merge step of our parallel divide-and-conquer algorithm takes $O(\log n)$ time and uses $n$ processors. This proves:

Theorem 4 Let $D_{1}, D_{2}, \ldots, D_{n}$ be a set of pseudo disks on the unit sphere. There is a CREW-PRAM algorithm that decides if the union of the interiors of these pseudo disks covers the unit sphere. If this is not the case, then the algorithm finds a point on the unit sphere that is not contained in the interior of any pseudo disk. The algorithm takes $O\left(\log ^{2} n\right)$ time and uses $n$ processors.

\section{Some related problems}

Until now, we considered maxmin-problems. In this section, we briefly discuss some dual versions.

Problem 3 Let $S$ be a set of $n$ points in $\mathbb{R}^{d}$, and let each point $p$ of $S$ have a weight $w(p)$, which is a positive real number. Compute an anchored ray $R$ for which $\max _{p \in S} w(p) \cdot d(p, R)$ is minimal.

As before, we can assume w.l.o.g. that all points have weight one. In [11], Lee and Wu show how to solve this problem in $O(n \log n)$ time when $d=2$. They state as an open problem to decide whether this is optimal.

We show how to solve Problem 3 for $d=3$. Let $B_{p}^{\delta}$ denote the ball with center $p$ and radius $\delta$. Then we want to compute the minimal real number $\delta \geq 0$ such that there is an anchored ray that intersects all balls $B_{p}^{\delta}, p \in S$. We find this minimal $\delta$ using the parametric search technique.

Let $\delta \geq 0$. We need sequential and parallel algorithms for deciding if there is an anchored ray that intersects all balls $B_{p}^{\delta}, p \in S$. Clearly, we do not have to consider those balls that contain the origin. Using the same approach as in Section 4, we arrive at the following problem: Given a set of at most $n$ disks on the unit sphere, decide if their intersection is empty. This intersection has combinatorial complexity $O(n)$. Moreover, it can be computed by basically the same approach as in Section 4.2.1. Hence, we get the following result.

Theorem 5 Let $S$ be a set of $n$ points in $\mathbb{R}^{3}$, and let each point $p$ of $S$ have a positive weight $w(p)$. In $O\left(n \log ^{4} n\right)$ time, we can compute an anchored ray $R$ for which $\max _{p \in S} w(p) \cdot d(p, R)$ is minimal.

Problem 4 Let $S$ be a set of $n$ points in $\mathbb{R}^{d}$, and let each point $p$ of $S$ have a weight $w(p)$, which is a positive real number. Compute a line l through the origin for which $\max _{p \in S} w(p) \cdot d(p, l)$ is minimal.

For $d=2$, this problem can be solved in $O(n \log n)$ time, which is optimal in the algebraic computation tree model. See [11]. The three-dimensional version seems to be much harder. Follert [6] solves this problem in $O\left(n \lambda_{6}(n) \log n\right)$ time. 
A symmetric slab is defined as the region between two parallel planes in $\mathbb{R}^{3}$ that are at the same distance from the origin. If we intersect a symmetric slab with the unit sphere, then we get a symmetric slab on the unit sphere. A natural approach to solve the three-dimensional version of Problem 4 is to use the parametric search technique. Then we have to design sequential and parallel algorithms for the following decision problem: Given a set of $n$ symmetric slabs on the unit sphere, do they cover the unit sphere.

This decision problem resembles the following problem: Given a circle $C$ and a set of $n$ slabs, both in the plane, decide whether these slabs cover $C$. Gajentaan and Overmars [7] proved that this problem is $n^{2}$-hard, which indicates that it is probably very hard to find a subquadratic algorithm for it.

Open problem 1 Decide if the problem

Given a set of $n$ symmetric slabs on the unit sphere, do they cover the unit sphere,

is $n^{2}$-hard, or if it can be solved in subquadratic time.

Note that if this problem is $n^{2}$-hard, that then also the three-dimensional version of Problem 4 is $n^{2}$-hard.

Problem 5 Let $S$ be a set of $n$ points in $\mathbb{R}^{d}$, and let each point $p$ of $S$ have a weight $w(p)$, which is a positive real number. Compute a hyperplane $H$ through the origin for which $\max _{p \in S} w(p) \cdot d(p, H)$ is minimal.

Lee and $\mathrm{Wu}[11]$ proved an $\Omega(n \log n)$ lower bound for the planar version of this problem. Clearly, this implies the same lower bound for any dimension $d$.

We show how to solve the three-dimensional version of Problem 5 in $O(n \log n)$ time. We can assume w.l.o.g. that all points of $S$ have weight one.

Our problem is equivalent to that of computing the symmetric slab of minimal width that contains all points of $S$. Let $S^{\prime}:=S \cup-S$, where $-S:=\left\{\left(-p_{1},-p_{2},-p_{3}\right)\right.$ : $\left.\left(p_{1}, p_{2}, p_{3}\right) \in S\right\}$. For any plane $H$ through the origin, we have $H=-H$. Therefore,

$$
d(p, H)=d(-p,-H)=d(-p, H) .
$$

As a result, it suffices to solve our problem for the set $S^{\prime}$. Since this set is symmetric w.r.t. the origin, the width of the minimal symmetric slab containing $S^{\prime}$ is equal to the width of $S^{\prime}$, which is defined as the minimal width of any slab containing this set.

The best known algorithm for computing the width of an arbitrary set of $n$ points in $\mathbb{R}^{3}$ has running time $O\left(n^{17 / 11+\epsilon}\right)$, where $\epsilon$ is an arbitrarily small positive constant. (See Agarwal et al. [1].) In our case, however, the set of points has a special form. As we will see, this allows to compute the width of $S^{\prime}$ in $O(n \log n)$ time.

Houle and Toussaint [9] observed that the width of a set of points in $\mathbb{R}^{3}$ is the minimum distance between parallel planes of support passing through either an antipodal vertex-face pair or an antipodal edge-edge pair of the convex hull of the set.

It is not difficult to see that in order to compute the width of our set $S^{\prime}$, we only have to consider parallel planes of support passing through an antipodal vertex-face 
pair of the convex hull of $S^{\prime}$, and take the minimum distance between any such pair of planes. This minimum distance can be computed in $O(n \log n)$ time. (See [9].) Hence, we have proved the following result.

Theorem 6 Let $S$ be a set of $n$ points in $\mathbb{R}^{3}$, and let each point $p$ of $S$ have a positive weight $w(p)$. In $O(n \log n)$ time, we can compute a plane $H$ through the origin for which $\max _{p \in S} w(p) \cdot d(p, H)$ is minimal. This is optimal in the algebraic computation tree model.

\section{Acknowledgement}

The authors thank Prof. Hotz for posing the problems that were considered in this paper. They also thank Christine Rüb and Stefan Schirra for several helpful discussions.

\section{References}

[1] P.K. Agarwal, B. Aronov, and M. Sharir. Computing enveloped in four dimensions with applications. Proc. 10th Annual ACM Conf. on Comp. Geom., 1994, pp. 348358.

[2] P.K. Agarwal, A. Efrat, M. Sharir, and S. Toledo. Computing a segment center for a planar point set. J. Algorithms 15 (1993), pp. 314-323.

[3] P.K. Agarwal, M. Sharir, and P. Shor. Sharp upper and lower bounds for the length of general Davenport-Schinzel sequences. J. Combin. Theory, Ser. A 52 (1989), pp. 228-274.

[4] P.K. Agarwal, M. Sharir, and S. Toledo. Applications of parametric searching in geometric optimization. J. Algorithms 17 (1994), pp. 292-318.

[5] N. Amato and F. Preparata. The parallel $3 D$ convex hull problem revisited. Comp. Geom. \& Applications 2 (1992), pp. 163-173.

[6] F. Follert. Lageoptimierung nach dem Maximin-Kriterium. Master's Thesis. Department of Computer Science, Universität des Saarlandes, Saarbrücken, 1994.

[7] A. Gajentaan and M.H. Overmars. $n^{2}$-Hard problems in computational geometry. Tech. Report RUU-CS-93-15, Department of Computer Science, University of Utrecht, 1993.

[8] L. Guibas and M. Sharir. Combinatorics and algorithms of arrangements. In: New Trends in Discrete and Computational Geometry, Ed. J. Pach. Springer-Verlag, Berlin, 1993, pp. 9-36.

[9] M.E. Houle and G.T. Toussaint. Computing the width of a set. IEEE Trans. Pattern Anal. Mach. Intell., PAMI-10 (1988), pp. 761-765. 
[10] K. Kedem, R. Livne, J. Pach, and M. Sharir. On the union of Jordan regions and collision-free translational motion amidst polygonal obstacles. Discrete Comput. Geom. 1 (1986), pp. 59-71.

[11] D.T. Lee and Y.F. Wu. Geometric complexity of some location problems. Algorithmica 1 (1986), pp. 193-211.

[12] N. Megiddo. Applying parallel computation algorithms in the design of serial algorithms. J. ACM 30 (1983), pp. 852-865.

[13] A. Melkman and J. O'Rourke. On polygonal chain approximation. In: Computational Morphology (Ed. G.T. Toussaint), North-Holland, Amsterdam, 1988, pp. $87-95$.

[14] T. Ottmann, P. Widmayer, and D. Wood. A fast algorithm for Boolean mask operations. Comput. Vision Graph. Image Process. 30 (1985), pp. 249-286.

[15] F.P. Preparata and M.I. Shamos. Computational Geometry, an Introduction. Springer-Verlag, New York, 1985.

[16] C. Rüb. Computing intersections and arrangements for red-blue curve segments in parallel. Proc. 4th Canadian Conf. on Comp. Geom., 1992, pp. 115-120. 